OPEN ACCESS

Edited by: Brian Ahmer,

Ohio State University, USA

Reviewed by:

Andres Vazquez-Torres, University of Colorado Denver, USA José Alejandro Di Conza, UBA and UNL, Argentina

*Correspondence: Ya-Hong Liu lyh@scau.edu.cn Jian Sun

jiansun@scau.edu.cn

Received: 21 November 2016 Accepted: 31 January 2017

Published: 17 February 2017

Citation:

Chen Y, Hu D, Zhang Q, Liao X-P, Liu Y-H and Sun J (2017) Efflux Pump

Overexpression Contributes to

Tigecycline Heteroresistance in

Salmonella enterica serovar

Typhimurium

Front. Cell. Infect. Microbiol. 7:37. doi: $10.3389 /$ fcimb.2017.00037

\section{Efflux Pump Overexpression Contributes to Tigecycline Heteroresistance in Salmonella enterica serovar Typhimurium}

\author{
Yi Chen ${ }^{1,2}$, Daxing Hu ${ }^{1,2}$, Qijing Zhang ${ }^{3}$, Xiao-Ping Liao ${ }^{1,2}$, Ya-Hong Liu ${ }^{1,2 *}$ and Jian Sun ${ }^{1,2 *}$ \\ ${ }^{1}$ National Risk Assessment Laboratory for Antimicrobial Resistance of Animal Original Bacteria, College of Veterinary \\ Medicine, South China Agricultural University, Guangzhou, China, ${ }^{2}$ Guangdong Provincial Key Laboratory of Veterinary \\ Pharmaceutics Development and Safety Evaluation, South China Agricultural University, Guangzhou, China, ${ }^{3}$ Department of \\ Veterinary Microbiology and Preventive Medicine, lowa State University, Ames, IA, USA
}

Bacterial heteroresistance has been identified in several combinations of bacteria and antibiotics, and it complicated the therapeutic strategies. Tigecycline is being used as one of the optimal options for the treatment of infections caused by multidrugresistant Salmonella. This study investigated whether heterorresistance to tigecycline exists in a Salmonella enterica serovar Typhimurium strain harboring the oqxAB-bearing IncHI2 plasmid pHXY0908. MIC and population analyses were performed to evaluate population-wide susceptibility to tigecycline. The effects of efflux pumps on MIC levels were assessed using the efflux pump inhibitor Phe-Arg- $\beta$-naphthylamide, measuring intracellular tigecycline accumulation as well as mRNA levels of regulatory and efflux pump genes. DNA sequencing of regulatory regions were performed and plasmid curing from a resistant strain provided an appropriate control. Results showed that MICs of a parental strain with and without pHXY0908 as well as a plasmid-cured strain $14028 / \Delta$ p52 were $0.5,1$, and $1 \mu \mathrm{g} / \mathrm{mL}$, respectively. Population analysis profiling (PAP) illustrated that only the pHXY0908-containg strain was heteroresistant to tigecycline. A fraction of colonies exhibited stable profiles with 4- to 8-fold increases in MIC. The frequencies of emergence of these isolates were higher in the plasmid-containing strain pHXY0908 than either the parental or the 14028/ $\Delta$ p52 strain. Phe-Arg- $\beta$-naphthylamide addition restored tigecycline susceptibility of these isolates and intracellular tigecycline accumulation was reduced. Heteroresistant isolates of the strain containing pHXY0908 also had elevated expression of $\operatorname{acr} B$, $\operatorname{ram} A$, and oqxB. DNA sequencing identified numerous mutations in RamR that have been shown to lead to ramA overexpression. In conclusions, heteroresistance to tigecycline in Salmonella enterica serovar Typhimurium was manifested in a plasmid-bearing strain. Our results suggest that this phenotype was associated with overexpression of the AcrAB-TolC and OqxAB efflux pumps.

Keywords: tigecycline, Salmonella enterica serovar Typhimurium, heteroresistance, efflux pump, plasmid 


\section{INTRODUCTION}

Salmonella enterica serovar Typhimurium is an important zoonotic pathogen that leads to various infections in humans and the proliferation of multidrug-resistant isolates, especially those extended-spectrum $\beta$-lactamase-producing and fluoroquinolone-resistant strains are of major concerns (Fàbrega et al., 2008). Carbapenems and colistin were therapeutic options for treatment of severe infections caused by multidrug-resistant bacteria such as the Typhimurium serovar (Taneja and Kaur, 2016). However, the emergence of carbapenemase-producing and mcr-1-positive Salmonella has threatened the treatment options (Liu et al., 2016; Yao et al., 2016).

Tigecycline represents a new class of antibiotic agents, the glycylcyclines. This compound exhibits an extraordinarily broad-spectrum of activity against pathogens, including difficult-to-treat pathogens such as carbapenemase- or extended-spectrum $\beta$-lactamases-producing Gram-negative bacteria (Stein and Babinchak, 2013). Antimicrobial activity of tigecycline results from a stacking interaction with nucleobase C1054 within the decoding site of the ribosome and during decoding, tigecycline inhibits the initial codon recognition step of tRNA accommodation (Jenner et al., 2013). Tigecycline is readily transported into the cell and achieves high intracellular concentrations (Ong et al., 2005). This is a key factor when considering treatment of invasive infections caused by multidrug-resistant Salmonella (Tang et al., 2016).

Although tigecycline bypasses the classical resistance mechanisms that confer resistance to tetracyclines (Fluit et al., 2005), the compound is still vulnerable to chromosomally encoded efflux pumps (Keeney et al., 2007, 2008; Ruzin et al., 2007). Up-regulation of multidrug efflux pumps such as AcrAB in Escherichia coli (Keeney et al., 2008), Klebsiella pneumonia (Zhong et al., 2014), and Enterobacter cloacae (Keeney et al., 2007); AdeABC in Acinetobacter baumanni (Ruzin et al., 2007), and MexXY in Pseudomonas aeruginosa (Dean et al., 2003) have all been implicated in tigecycline resistance. An RND family efflux pump, OqxAB may also supplement tigecycline resistance in K. pneumonia (Zhong et al., 2014) and E. cloacae (Veleba et al., 2013). The AcrAB-TolC efflux pump transports numerous structurally unrelated compounds (Du et al., 2014). Its expression is modulated precisely by its local repressor, AcrR, as well as by global transcriptional regulators of the AraC family, RamA, MarA, SoxS, and Rob (Nikaido et al., 2008; Kehrenberg et al., 2009; Pérez et al., 2012). Mutations in acrR cause the loss of its repressor function, resulting in $a c r A B$ overexpression. In addition, mutations in $\operatorname{ram} R, \operatorname{mar} R$, and $\operatorname{sox} R$ activate $\operatorname{ram} A$, mar $A$, and soxS, leading to the upregulation of the $\operatorname{acr} A B$ efflux pump.

Heteroresistance is the presence of a subpopulation of less susceptible bacteria in a population of fully susceptible bacteria (El-Halfawy and Valvano, 2015). This phenomenon was first described in Staphylococcus aureus in 1970 (El-Halfawy and Valvano, 2015) and has been widely investigated. Distinct from the persistence which was linked to phenotypic switching between growing cells and persister cells having reduced growth rates and cells regrown from them remain sensitive to the antibiotic (Balaban et al., 2004), heteroresistance means the coexistence of resistant and susceptible bacterial cells in the same culture (El-Halfawy and Valvano, 2015). Population analysis profiling (PAP) is the conventional method for determining heteroresistance (El-Halfawy and Valvano, 2015). Bacterial antibiotic resistance can be either intrinsic or acquired and these are equally applicable to heteroresistance (El-Halfawy and Valvano, 2015). Heteroresistance can also be an intermediate stage for completely changing from susceptibility to resistance upon exposure of bacteria to an antibiotic (Morand and Mühlemann, 2007; Falagas et al., 2008).

Clinical isolates of Salmonella resistant to tigecycline have now been reported (Hentschke et al., 2010). Non-typhoidal Salmonella has a wide host specificity and global burden compared to typhoidal Salmonella (Smith et al., 2016). Up to this point, there have been no investigations on tigecycline heteroresistance in Salmonella enterica serovar Typhimurium. Thus the main purpose of this study was to investigate whether heteroresistance to tigecycline exists in Salmonella enterica serovar Typhimurium 14028 and 14028/pHXY0908 (14028 harboring oq $x A B$-bearing IncHI2 plasmid pHXY0908) and to determine molecular mechanisms responsible for tigecycline heteroresistance. 14028/pHXY0908 was obtained by electroporation of a transferable IncHI2-type plasmid pHXY0908 (KM877269) carrying the quinolone resistance determinate oqxAB from S. Typhimurium-147 into Salmonella enterica serovar Typhimurium 14028. The multidrug-resistant IncHI2 plasmid pHXY0908 (GenBank accession number KM877269) from $S$. Typhimurium has been involved in the spread of oq $x A B$ in food-producing animals in China (Li et al., 2013).

\section{MATERIALS AND METHODS}

\section{Strains and Antibiotic Susceptibility Testing}

The bacterial strains and plasmids used in this study are listed in Table 1. Typhimurium strains were routinely propagated in Mueller-Hinton (MH) or Luria-Bertani (LB) medium. The MIC of tigecycline was determined by standard broth microdilution methods according recommendations of the Clinical and Laboratory Standards Institute (M100-S25). All media were freshly prepared $(<12 \mathrm{~h})$ in order to minimize the oxidative degradation of tigecycline (Hope et al., 2005). The breakpoint criteria used to determine tigecycline phenotype was based on the United States Food and Drug Administration breakpoint criteria [ $\leq 2 \mathrm{mg} / \mathrm{L}$ (susceptibility), $4 \mathrm{mg} / \mathrm{L}$ (intermediate), and $\geq 8 \mathrm{mg} / \mathrm{L}$ (resistance)]. E. coli ATCC 25922 was included and used as a reference strain.

\section{Population Analysis Profiling (PAP)}

PAP was conducted as previously described (Hung et al., 2012). Briefly, $40 \mu \mathrm{L}$ of overnight culture was subcultured in $4 \mathrm{~mL}$ of LB broth, and grown to late logarithmic phase $\left(\mathrm{OD}_{600}=\right.$ $0.3-0.4)$. Dilutions of $10^{-2}-10^{-6}$ in $0.85 \%$ saline were prepared, and $100 \mu \mathrm{L}\left(\sim 10^{8}\right.$ bacterial $\left.\mathrm{CFU}\right)$ was spread onto $\mathrm{MH}$ agar plates containing tigecycline in serial dilutions at concentrations ranging from 0.125 to $16 \mu \mathrm{g} / \mathrm{mL}(0.5$ to $16 \times \mathrm{MIC})$. A dilution of $10^{-6}$ was deposited onto $\mathrm{MH}$ plates lacking antibiotic for 
TABLE 1 | Strains and plasmid used in this study.

\begin{tabular}{|c|c|}
\hline Strain or plasmid & Description \\
\hline \multicolumn{2}{|l|}{ S. TYPHIMURIUM } \\
\hline 14028 & ATCC 14028 \\
\hline 14028\#2 & Derived from 14028 with ramR mutation \\
\hline 14028\#3 & Derived from 14028 with ramR mutation \\
\hline 14028/pHXY0908 & Derivative of 14028 harboring plasmid pHXY0908 \\
\hline 14028/p\#36 & $\begin{array}{l}\text { Heteroresistant isolate of } 14028 / \mathrm{pHXY0908} \text { with ramR } \\
\text { mutation }\end{array}$ \\
\hline 14028/p\#52 & $\begin{array}{l}\text { Heteroresistant isolate of } 14028 / \mathrm{pHXY0908} \text { with ramR } \\
\text { mutation }\end{array}$ \\
\hline $14028 / \Delta p 52$ & Plasmid-cured strain of 14028/p\#52 \\
\hline 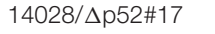 & Resistant isolate of $14028 / \Delta \mathrm{p} 52$ \\
\hline $14028 / \Delta \mathrm{p} 52 \# 18$ & Resistant isolate of $14028 / \Delta \mathrm{p} 52$ \\
\hline \multicolumn{2}{|l|}{ Plasmid } \\
\hline pHXY0908 & $\begin{array}{l}\text { An IncHI2-type plasmid containing oqXAB, sul, aphA1, } \\
\text { aadA1, cmIA, aadA2, floR, aac }\left(3^{\prime}\right)-I V, \operatorname{aac}\left(6^{\prime}\right)-I b-C r \text {, } \\
\text { blaOXA-1, catB3, qacEdelta1 (GenBank accession } \\
\text { number KM877269) }\end{array}$ \\
\hline
\end{tabular}

determination of colony number after $48 \mathrm{~h}$ at $37^{\circ} \mathrm{C}$. The frequency of appearance of heteroresistant subpopulations was calculated by dividing the number of colonies that grew on antibiotic-containing plates $(4-16 \times \mathrm{MIC})$ by the number that grew on antibiotic-free plates (Morand and Mühlemann, 2007). The analysis was performed three times and the mean values of viable CFU were calculated and plotted on a semilogarithmic graph.

\section{Luria-Delbrück Fluctuation Analysis and Stability of Resistance Profiles}

To investigate whether the resistant subpopulations were derived from pre-adapted antibiotic-resistant cells that initially existed in the culture, Luria-Delbrück fluctuation analysis was performed as previously described (Sánchez-Romero and Casadesus, 2014). Briefly, a small number of cells were used to inoculate parallel tubes containing LB broth and were then grown to saturation to obtain equal cell densities. One hundred microliters of overnight samples $\left(\sim 2 \times 10^{8}\right.$ cells $)$ from individual subcultures were separately plated onto LB agar plates containing tigecycline at concentrations of $5 \times$ MIC or $2.5 \times$ MIC. Simultaneously, samples containing the same amount of bacteria from single bacterial cultures were plated onto parallel plates lacking antibiotic. Colonies were counted after $48 \mathrm{~h}$ incubation. The concentration of tigecycline was selected to suppress sensitive cells in bacterial cultures and allow the growth of subpopulations containing high-level resistance that was judged by the results of MIC and PAP testing.

Survivors picked up from the drug-containing plates were determined susceptibility to tigecycline by an agar dilution method according to CLSI recommendation M100-S25. After seven daily subcultures in antibiotic-free medium, the tigecycline MICs were re-tested to check the stability of the resistant phenotypes. To determine whether efflux activity was responsible for reduced susceptibility to tigecycline, 2-fold serial broth microdilutions in 96-well plates in the presence or absence of $20 \mu \mathrm{g} / \mathrm{mL}$ of the efflux pump inhibitor phenylalanine-arginine $\beta$ naphthylamide $(\mathrm{PA} \beta \mathrm{N})$ dihydrochloride (Sigma, St. Louis, USA). Both unexposed parental strains and survivors showing stable profiles with 4- to 8-fold increases in MICs were chosen for further study. These selections were performed in triplicate and experiments were repeated twice. If the MIC values decreased 4 -fold or greater upon exposure to $\mathrm{PA} \beta \mathrm{N}$, efflux activity was judged as high. In order to explore the possibility of cross heteroresistance between unrelated antibiotics, susceptibility to ciprofloxacin was also determined for heteroresistant colonies.

\section{Accumulation of Tigecycline}

Efflux activity of selected isolates was further evaluated by determining the accumulation of tigecycline (inversely proportional to efflux activity) according to the method of ciprofloxacin accumulation (Sun et al., 2011). A liquid chromatography assay was used to determine tigecycline concentrations (Li et al., 2004). Differences in drug accumulation at steady-state between randomly selected survivors and the parental strain 14028 (see results) were analyzed for statistical significance using a two-tailed Student's $t$-test. $P<0.05$ was taken as significant. Data shown are means of at least three independent technical replicates $( \pm$ SEM).

\section{Quantitative Real-Time Pcr (qRT-PCR) Analysis}

The expression levels of marA, soxS, rob, and $\operatorname{ram} A$ regulator genes and efflux components $a c r A$, $a c r B$, tolC, and $\operatorname{oq} x B$ were assessed using qRT-PCR. Bacteria were grown to midlogarithmic phase and total RNA was extracted by using Trizol reagent (Invitrogen, Carlsbad, CA). RNA yield and quality were measured using a BioPhotometer plus instrument (Eppendorf, Shanghai, China) and cDNA was synthesized from $0.5 \mathrm{mg}$ RNA templates using a PrimeScript RT reagent Kit with gDNA Eraser (Perfect Real Time) according to the manufacturer's instructions (TaKaRa, Dalian, China). qRT-PCR was carried out in an IQ5 thermal cycler (Bio-Rad, Hercules, CA) using SYBR ${ }^{\circledR}$ Premix Ex Taq (TaKaRa, Dalian, China). Primers are listed in the Table S1. PCR conditions were as previously described(Abouzeed et al., 2008; Kehrenberg et al., 2009; Zheng et al., 2009; Wong et al., 2015). qRT-PCR experiments were performed in triplicate using different RNA preparations for confirmation of the results. The $2^{-\Delta \Delta C t}$ method was used to calculate fold changes of mRNA levels of target genes in selected survivors compared with those observed in their respective parental strains (strains 14028, 14028/pHXY0908, and 14028/ $\Delta$ p52). Expression levels of all pump genes and pump regulators of parental strains were designated to be 1 . Only genes whose ratios were $\geq 2$ fold changes (either increased or decreased) were considered statistically significant (Zheng et al., 2011).

\section{DNA Sequence Analysis}

To detect mutations in regions known to be involved in the regulation of the efflux pump AcrAB-TolC, we amplified acrR, soxR, marR, and ramR using primers and PCR conditions as described previously (Olliver et al., 2004; Abouzeed et al., 2008). 
The PCR products were sent for DNA sequencing (company and location). Sequences were compared with those from the GenBank nucleotide database using the BLAST (Basic Local Alignment Search Tool) algorithm (http://www.ncbi.nlm.nih. gov/BLAST).

\section{Plasmid Curing}

To confirm the role of genes on the plasmid in tigecycline heteroresistance, the cure of plasmid from a heteroresistant isolate 14028/p\#52 was conducted using sodium dodecyl sulfate (SDS) as previously described (Akiyama et al., 2013). Briefly, an overnight culture was diluted 100 -fold in LB broth containing $10 \%$ SDS and then was incubated overnight at $42^{\circ} \mathrm{C}$. Bacterial suspensions were streaked onto LB agar and individual colonies were replica plated onto LB agar plates with or without olaquindox $(50 \mu \mathrm{g} / \mathrm{mL})$. To verify the plasmid loss, PCR amplification of the $o q x B$ gene was performed for those colonies that did not grow in olaquindox. The cured strain was named $14028 / \Delta \mathrm{p} 52$. Heteroresistance to tigecycline of $14028 / \Delta \mathrm{p} 52$ were characterized and experiments were conducted as those used for 14028 and 14028/pHXY0908.

\section{RESULTS}

\section{Acquiring an oqxAB-Bearing Plasmid pHXY0908 makes Salmonella Enterica Serovar Typhimurium Heteroresistant to Tigecycline}

The tigecycline MICs of the 14028, 14028/pHXY0908, and $14028 / \Delta$ p52 strains did not show significant differences and were $0.5,1$, and $1 \mu \mathrm{g} / \mathrm{mL}$, respectively. However, in strain 14028/pHXY0908, the PAP curve indicated the presence of subpopulations with high-level resistance to tigecycline at frequencies ranging from $10^{-5}$ to $10^{-8}$. Conversely, subpopulations of 14028 and $14028 / \Delta$ p52 did not show this extent of resistance and grew to less than four times their MICs (Figure 1).

\section{pHXY0908 Increases the Frequency of Emergence of Strains with Reduced Susceptibility}

Luria-Delbrück fluctuation analysis indicated that bacteria survived the selection process at relatively low frequencies $\left(\leq 10^{-7}\right.$; Tables S2-S4). The strain 14028/pHXY0908 survivors were cells that were present in the starting bacterial culture indicating no selection occurred during this experiment. MIC of isolates directly picked from drug-containing plates showed widely distributed values (Figure 1S).

We re-tested susceptibility of these colonies by subculturing in antibiotic-free medium. A substantial number of these strains returned to tigecycline susceptibility (Figure $1 S)$. Only $4(4 / 43)$, $21(21 / 55)$, and $9(9 / 36)$ isolates of $14028,14028 / \mathrm{pHXY} 0908$, and $14028 / \Delta$ p52 respectively, showed stable profiles with 4 to 8 -fold MIC increases.

The frequency of emergence of these isolates was significantly higher in strain 14028/pHXY0908 than in either 14028 or

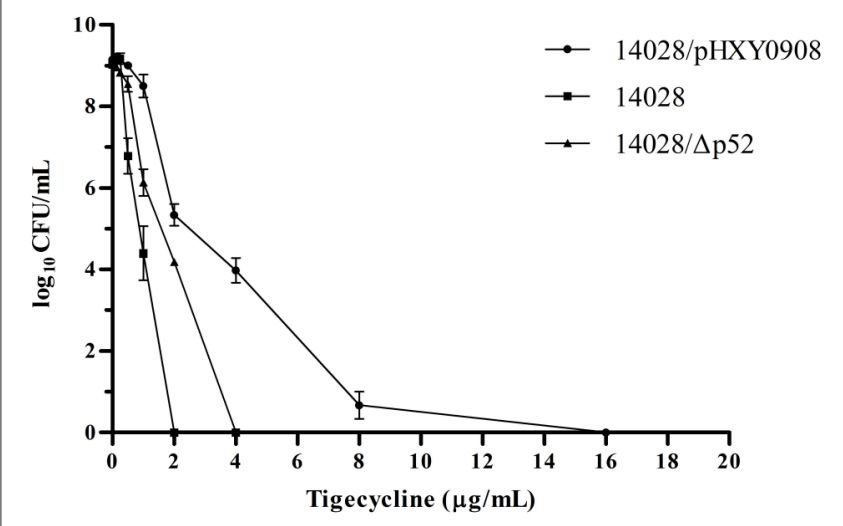

FIGURE 1 | Susceptibility of S. Typhimurium14028, 14028/pHXY0908 and $14028 / \Delta \mathrm{p} 52$ to tigecycline as demonstrated by population analysis. The $x$ axis indicates the tigecycline concentration in micrograms per milliliter used to select supopulations with higher tigecycline-resistance levels, and on the $y$ axis, the frequency of bacterial cells is given as the logarithm to the base 10 of cfu per milliliter.

$14028 / \Delta \mathrm{p} 52(P<0.05)$. Ciprofloxacin susceptibility of 21 heteroresistant isolates of $14028 / \mathrm{pHXY} 0908$ were tested and exhibited 4- to 8 -fold increases in MICs $(1-2 \mu \mathrm{g} / \mathrm{mL}$ for heteroresistant isolates; $0.25 \mu \mathrm{g} / \mathrm{mL}$ for $14028 / \mathrm{pHXY0908)}$ (detailed data not shown).

\section{Tigecycline Susceptibility Was Reversed by Adding Efflux Pump Inhibitor (PA $\beta N$ )}

As efflux pumps are implicated in tigecycline resistance in Salmonella, we examined whether efflux pumps were also associated with tigecycline heteroresistance. The addition of the efflux pump inhibitor PA $\beta N$ reduced MIC values 2- to 64-fold (Table S5). This data indicated that efflux pumps are involved in heteroresistance.

\section{Less Accumulation of Tigecycline in Strains with Reduced Susceptibility}

Since the intracellular accumulation of drugs were inversely proportional to efflux activity, we measured concentrations of tigecycline in selected survivors and parental strain. Results showed that the steady-state tigecycline concentrations in two plasmid containing strains (14028/p\#36 and 14028/p\#52) were 2 - and 3 -fold less than the parental strain $14028(P<0.001)$. Reduced tigecycline accumulation was also observed in 14028\#2 $(P<0.05), 14028 \# 3(P<0.05), 14028 / \Delta \mathrm{p} 52 \# 17(P<0.01)$, and $14028 / \Delta \mathrm{p} 52 \# 18(P<0.05)$ (Figure 2).

\section{Upregulation of Pump Genes and Regulators in Strains with Reduced Susceptibility}

To further confirm that efflux pumps were responsible for the reduced tigecycline susceptibility and accumulation, we selected survivors and determined the relative mRNA levels of the efflux pump genes $a c r B$ and $o q x B$. The levels of both genes in all strains 


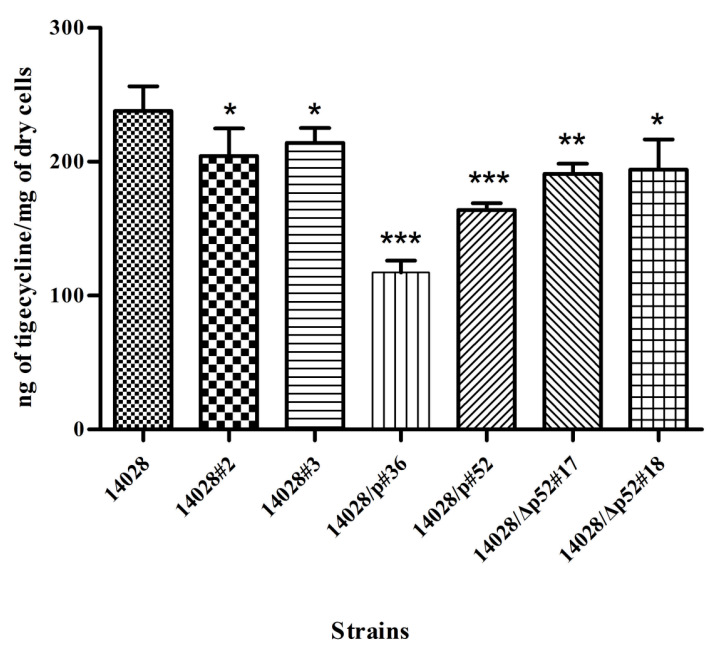

FIGURE 2 | Accumulation of tigecycline in isolates relative to strain 14028. The data shown are the means of two biological replicates. ${ }^{*} P<0.05$; ${ }^{\star \star} P<0.01 ;{ }^{\star * \star} P<0.001$

were 2- to 5 -fold greater than controls. This trend was also seen with the global regulator $\operatorname{ram} A$. Interestingly, in two isolates the increases were 28.5- and 77-fold (Figure 3 and Figure 2S). Upregulation of pump genes $a c r A$, tolC, and pump regulator marA were observed in all of tested isolates. Strain 14028/p\#36 also showed increased expression of soxS while rob expression was similar to those of the parental strains.

Interestingly, curing the plasmid in 14028/p\#52 decreased the expression of $a c r A$ by $-5.45- \pm 0.70$-fold and $a c r B$ by $-3.47- \pm$ 0.57 -fold (data not shown) and decreased the tigecycline MIC to $1 \mu \mathrm{g} / \mathrm{mL}$.

\section{Mutations in ramR Gene Leads to Overexpression of ramA and acrAB}

To investigate causes of the increased expression of $\operatorname{ram} A$ and $a c r A B$, DNA sequencing of the regulatory regions was performed. Analyzing of $\mathrm{ramR}$ gene detected a point mutation, ACC $\rightarrow$ CCC, at position 52 leading to the amino acid substitution of threonine for proline $(\mathrm{T} 18 \mathrm{P})$ in the resistant isolates $14028 / \mathrm{p} \# 36$, $14028 / \mathrm{p} \# 52,14028 / \Delta \mathrm{p} 52 \# 17$, and $14028 / \Delta$ p52\#18 (Table 2). In $14028 \# 2$, a mutation at amino acid 39 of RamR (V39A) was identified. An insertion of $1 \mathrm{bp}$ (A) located at nucleotide position 63 within the ramR gene was identified in $14028 \# 3$. This insertion led to occurrence of a premature stop codon (R21Stop). All of tested strains were confirmed to have wild type $\operatorname{acr} R$, soxR, and marR sequences.

\section{DISCUSSION}

Heteroresistance describes the coexistence of susceptible and resistant isolates in an otherwise genetically identical population (Hung et al., 2012). This study illustrates that heteroresistance to tigecycline in $S$. Typhimurium harboring an oq $x A B$ bearing multidrug resistance plasmid (Table 2). The increase in resistant subpopulations was greatest in strain 14028/pHXY0908 compared to strains 14028 and $14028 / \Delta$ p52. It also exhibited a heteroresistance profile while a homogeneous response to tigecycline was observed in parental strain 14028. Interestingly, although $14028 / \Delta$ p52 and 14028/pHXY0908 exhibited the same susceptibility to tigecycline by the broth microdilution method, subpopulations of $14028 / \triangle \mathrm{p} 52$ were unable to survive at $4 \times$ MIC, similar to 14028 . This indicated that the oqxAB-bearing plasmid is the reason for the heteroresistant phenotype of 14028/pHXY0908.

Subpopulations with high-level resistance to tigecycline occurred at relatively low frequencies $\left(10^{-5}-10^{-8}\right)$ and MICvalues $(1 \mu \mathrm{g} / \mathrm{mL})$ were below the susceptibility breakpoint $(2 \mu \mathrm{g} / \mathrm{mL})$. This indicates that the CLSI reference methods for antimicrobial susceptibility testing using an inoculum of $5 \times 10^{5} \mathrm{CFU} / \mathrm{mL}$ would classify such heteroresistant isolates as susceptible (Gomes et al., 2015). A similar assessment of susceptibility levels were found in a previous study that identified heterogeneous growth to carbapenems in $K$. pneumoniae carbapenemase (KPC)-producing Enterobacteriaceae (Pournaras et al., 2010). Accordingly, evaluating results of susceptibility testing should include determination of heteroresistant isolates.

In the present study, a resistance profile was maintained in the absence of selection stress in $S$. Typhimurium. The frequencies of emergent isolates showing stable profiles (4- to 8-fold increase in MIC) were significantly higher in 14028/pHXY0908 than in 14028 and $14028 / \Delta$ p52. Again, this suggests that plasmid pHXY0908 facilitates the appearance of tigecycline-nonsusceptible isolates. Heteroresistant isolates of 14028/pHXY0908 also had a decreased susceptibility to ciprofloxacin. This indicated the presence of a mechanism leading to crossheteroresistance to different antibiotic classes (Hornsey et al., 2010).

Previous study has indicated that upregulation of the AcrAB efflux pump upon exposure to ciprofloxacin caused crossresistance to tigecycline (Hornsey et al., 2010). This occurrence is of great concern because of the greater probability to induce cross-resistance to new antibiotics with the application of empirical therapies. Overexpression of efflux pump can also lead to tigecycline resistance in $S$. aureus and, furthermore, that the acquisition of this resistance may be associated with reduced susceptibility to vancomycin (Herrera et al., 2016). After addition of PA $\beta N$, reduction of MIC values was observed for all resistant isolates that indirectly proves that efflux pump over-expression contributed to reduced tigecycline susceptibility. Furthermore, we directly confirmed efflux activity by determining the accumulation of intracellular of tigecycline. Therefore, this was an effective mechanism for heteroresistance or non-susceptibility in $S$. Typhimurium pertaining to activity of tigecycline.

A recent study has been sought to elucidate the roles of Salmonella multidrug efflux pumps and AcrAB regulators in tigecycline resistance, and results obtained showed that deletion of $\operatorname{acr} A B$ led to strains with significantly increased susceptibility to tigecycline and plasmids carrying the acr $A B$ restored increased susceptibility of the $a c r A B$-deleted mutant (Horiyama et al., 2011). That study demonstrated that reduced 

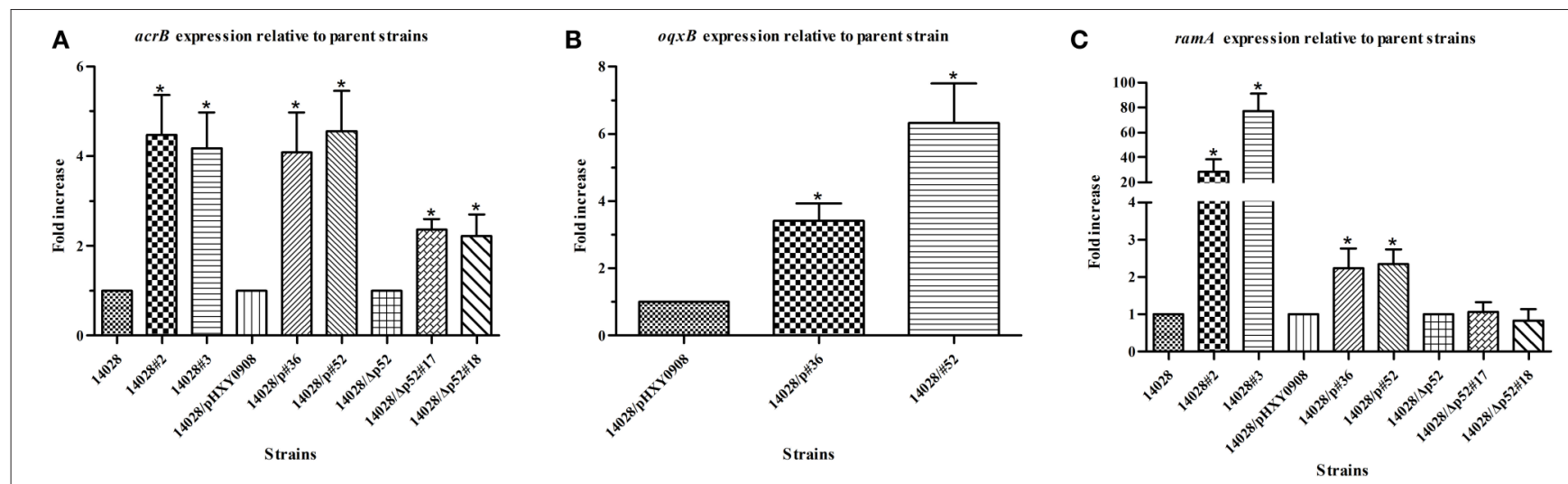

FIGURE 3 | Comparison of relative expression levels of acrB (A), oqxB (B) and ramA (C) in isolates 14028\#2, 14028\#3, 14028/p\#36, 14028/p\#52, $14028 / \Delta \mathrm{p} 52 \# 17$ and 14028/ $\Delta$ p52\#18 compared with their respective parental strains $14028,14028 / \mathrm{pHXY0908}$ and $14028 / \Delta \mathrm{p} 52$, and oqxB (B) in isolates 14028/p\#36 and 14028/p\#52 compared with 14028/pHXY0908. Expression level of acrB, oqxB, and ramA of parental strains were designated to be $1 .{ }^{*} P<0.01$.

TABLE 2 | Characteristics of Salmonella strains identified in this study.

\begin{tabular}{|c|c|c|c|c|c|c|c|}
\hline Strains & Heteroresistance ${ }^{a}$ & $\begin{array}{l}\text { MIC range } \\
(\mu \mathrm{g} / \mathrm{mL})^{b}\end{array}$ & $\begin{array}{c}\text { Stability rate } \\
(\%)\end{array}$ & $\begin{array}{c}\text { Inhibited by } \\
\text { PA } \beta N^{d}\end{array}$ & $\begin{array}{l}\text { Intracellular } \\
\text { accumulatione }\end{array}$ & $\begin{array}{l}\text { Expression } \\
\text { level of acrB, } \\
o q x B, \operatorname{ram} A^{f}\end{array}$ & $\begin{array}{l}\text { Nucleotide } \\
\text { Mutation (amino } \\
\text { acid change) }^{\mathrm{g}}\end{array}$ \\
\hline 14028/pHXY0908 & + & $1-8$ & $21 / 55$ (38.18\%) & Yes & * & $\uparrow$ & A52C (T18P) ${ }^{g 3}$ \\
\hline $14028 / \Delta p 52$ & - & $1-8$ & $9 / 36(25 \%)$ & Yes & $\star \star$ & $\uparrow$ & A52C (T18P)g3 \\
\hline
\end{tabular}

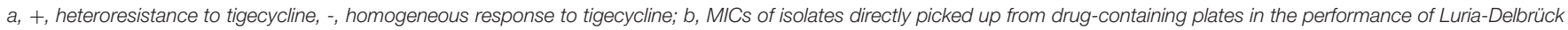

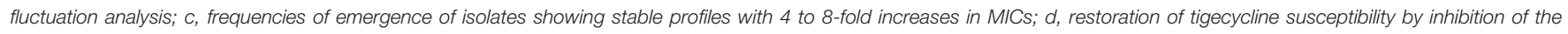

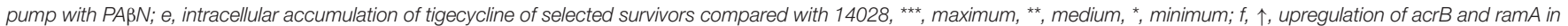

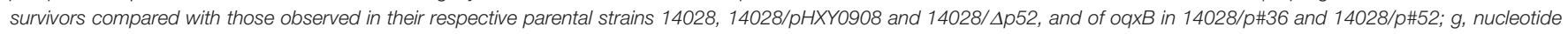

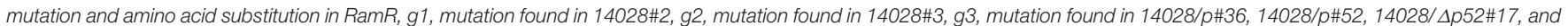
14028/4p52\#18, ins, insertion.

susceptibility to tigecycline might develop as a result of increased expression of efflux pump AcrAB in Salmonella enterica (Horiyama et al., 2011). Up-regulation of AdeABC, another resistance-nodulation-division efflux pump, has been proved resulting in decreased susceptibility to tigecycline in $A$. calcoaceticus-A. baumannii (Ruzin et al., 2007). In the present study, overexpression of $a c r A B$ in $14028 / p \# 36$ and 14028/p\#52 were observed indicating that an active efflux system was closely associated with heteroresistance to tigecycline. Consistent with the published data that $A c r A B$ confers resistance to tigecycline (Horiyama et al., 2011), upregulation of AcrAB was also observed in $14028 / \Delta \mathrm{p} 52 \# 17$ and $14028 / \Delta \mathrm{p} 52 \# 18$. In addition, overexpression of the $o q x B$ gene in both heteroresistant isolates indicated that efflux pump OqxAB might play a role in mediating tigecycline heteroresistance in $S$. Typhimurium strains.

Up-regulation of global regulators $\operatorname{ram} A, \operatorname{mar} A$, and soxS, which activate the AcrAB efflux pump, were also observed in isolates tested individually or as a group. Additionally, in strains $14028 / \mathrm{p} \# 36,14028 / \mathrm{p} \# 52,14028 / \Delta \mathrm{p} 52 \# 17$, and $14028 / \Delta \mathrm{p} 52 \# 18$ we found an amino acid change (T18P) in $\operatorname{ram} R$, which has been reported to play a role in the upregulation of RamA and AcrAB (Abouzeed et al., 2008). This resulted in an efflux-mediated MDR phenotype in S. Typhimurium.

Overexpression of $\operatorname{ram} A$ and inactivation of $\operatorname{ramR}$ involved in AcrAB-dependent tigecycline resistance in Salmonella have been suggested previously (Horiyama et al., 2011). In the current study, these mutations in the $\operatorname{ram} R$ gene led to upregulation of $\operatorname{ram} A$ resulting in tigecycline heteroresistance in our tested isolates. In $K$. pneumoniae, $\operatorname{oq} x A B$ is downregulated by a repressor $(o q x R)$ in the presence of a positive level of control via RamA, SoxS, and RarA (Veleba et al., 2012; JiménezCastellanos et al., 2016). Loss of OqxR repressor function was observed from the plasmid-borne oqxAB gene (Wong et al., 2015). Thus, ramA overexpression seemed to upregulate the oqxAB efflux pump in strains 14028/p\#36 and 14028/p\#52. However, further work is necessary to explore whether RamA can control the expression of the plasmid-encoded oq $x A B$ gene or whether RamA-mediated efflux pump overexpression can increase plasmid-mediated resistance (Jiménez-Castellanos et al., 2016).

Interestingly, when we cured the oq $x A B$-bearing plasmid from a heteroresistant isolate (14028/p\#52), restoration of tigecycline susceptibility and downregulation of pump genes $\operatorname{acr} A$ and 
acrB were observed (data not shown). After we have test the susceptibility of a cured strain in which the plasmid has been reintroduced and the MIC was $8 \mu \mathrm{g} / \mathrm{mL}$, indicating that approach used for curing the plasmid would not affect the susceptibility to tigecycline (data not shown). Thus, we hypothesized that in addition to the global transcriptional regulator RamA, genes on the plasmid might be also involved in the regulation of AcrABTolC, contributing to tigecycline heteroresistance. Deciphering potential mechanisms by which the oq $x A B$-bearing plasmid participates in the regulation of AcrAB-TolC pump are under the investigation in our laboratory.

It remains controversial whether heteroresistance is directly linked to treatment failure (Moore et al., 2003; Falagas et al., 2008; Deresinski, 2009; Lee et al., 2011) even though there is clinical evidence that tigecycline monotherapy is associated with a high mortality rate. Results obtained in the present study suggested that the rapid induction of efflux pumps AcrAB-TolC and OqxAB upon a single exposure to tigecycline in a heteroresistant Salmonella strain in vitro might leads to tigecycline resistance. Thus, heteroresistance should be taken into account when tigecycline treatment is used to avoid inadvertently inducing bacterial resistance. Appropriate detection methods are required to guide therapeutic decision-making.

Heteroresistance to tigecycline has not been previously reported so it is unclear whether there are other heteroresistant species and what clinical significance this may have. Systematic investigations on tigecycline heteroresistance in other pathogens and the relationship between heteroresistance and treatment failure are of practical importance.

In summary, the observations of present study suggested that an epidemic oqxAB-bearing IncHI2-type plasmid might engender a heteroresistant phenotype to $S$. Typhimurium

\section{REFERENCES}

Abouzeed, Y. M., Baucheron, S., and Cloeckaert, A. (2008). ramR mutations involved in efflux-mediated multidrug resistance in Salmonella enterica serovar Typhimurium. Antimicrob. Agents Chemother. 52, 2428-2434. doi: 10.1128/AAC.00084-08

Akiyama, T., Presedo, J., and Khan, A. A. (2013). The tetA gene decreases tigecycline sensitivity of Salmonella enterica isolates. Int. J. Antimicrob. Agents 42, 133-140. doi: 10.1016/j.ijantimicag.2013.04.017

Balaban, N. Q., Merrin, J., Chait, R., Kowalik, L., and Leibler, S. (2004). Bacterial persistence as a phenotypic switch. Science 305, 1622-1625. doi: 10.1126/science.1099390

Dean, C. R., Visalli, M. A., Projan, S. J., Sum, P. E., and Bradford, P. A. (2003). Efflux-mediated resistance to tigecycline (GAR-936) in Pseudomonas aeruginosa PAO1. Antimicrob. Agents Chemother. 47, 972-978. doi: 10.1128/AAC.47.3.972-978.2003

Deresinski, S. (2009). Vancomycin heteroresistance and methicillin-resistant Staphylococcus aureus. J. Infect. Dis. 199, 605-609. doi: 10.1086/596630

Du, D., Wang, Z., James, N. R., Voss, J. E., Klimont, E., Ohene-Agyei, T., et al. (2014). Structure of the AcrAB-TolC multidrug efflux pump. Nature 509, 512-515. doi: 10.1038/nature13205

El-Halfawy, O. M., and Valvano, M. A. (2015). Antimicrobial heteroresistance: an emerging field in need of clarity. Clin. Microbiol. Rev. 28, 191-207. doi: 10.1128/CMR.00058-14

Fàbrega, A., Sánchez-Céspedes, J., Soto, S., and Vila, J. (2008). Quinolone resistance in the food chain. Int. J. Antimicrob. Agents 31, 307-315. doi: 10.1016/j.ijantimicag.2007.12.010 against tigecycline. This phenomenon was probably related to overexpression of multidrug resistant efflux pumps AcrAB-TolC and OqxAB.

\section{AUTHOR CONTRIBUTIONS}

YC and JS designed the experiments, analyzed data, and wrote the manuscript. $\mathrm{YC}$ and $\mathrm{DH}$ performed the experiments and collected the data. YC, QZ, XL, and JS prepared the manuscript. All authors have contributed to, seen and approved the manuscript.

\section{ACKNOWLEDGMENTS}

This work was supported by the Funds for International Cooperation and Exchange of the National Natural Science Foundation of China (Grant No 31520103918), the National Natural Science Foundation and Natural Science Foundation of Guangdong Province, China (U1201214), the Programs of Changjiang Scholars and Innovative Research Team in University of Ministry of Education of China (IRT13063), Pearl River S\&T Nova Program of Guangzhou (Grant No. 201610010036), and National Natural Science Foundation of China (Grant No. 31402247).

\section{SUPPLEMENTARY MATERIAL}

The Supplementary Material for this article can be found online at: http://journal.frontiersin.org/article/10.3389/fcimb. 2017.00037/full\#supplementary-material

Falagas, M. E., Makris, G. C., Dimopoulos, G., and Matthaiou, D. K. (2008) Heteroresistance: a concern of increasing clinical significance? Clin. Microbiol. Infect. 14, 101-104. doi: 10.1111/j.1469-0691.2007.01912.x

Fluit, A. C., Florijn, A., Verhoef, J., and Milatovic, D. (2005). Presence of tetracycline resistance determinants and susceptibility to tigecycline and minocycline. Antimicrob. Agents Chemother. 49, 1636-1638. doi: 10.1128/AAC.49.4.1636-1638.2005

Gomes, D. M., Ward, K. E., and LaPlante, K. L. (2015). Clinical implications of vancomycin heteroresistant and intermediately susceptible Staphylococcus aureus. Pharmacother. 35, 424-432. doi: 10.1002/phar.1577

Hentschke, M., Christner, M., Sobottka, I., Aepfelbacher, M., and Rohde, H. (2010). Combined ramR mutation and presence of a Tn1721-associated tet(A) variant in a clinical isolate of Salmonella enterica serovar Hadar resistant to tigecycline. Antimicrob. Agents Chemother. 54, 1319-1322. doi: 10.1128/AAC.00993-09

Herrera, M., Di Gregorio, S., Fernandez, S., Posse, G., Mollerach, M., and Di Conza, J. (2016). In vitro selection of Staphylococcus aureus mutants resistant to tigecycline with intermediate susceptibility to vancomycin. Ann. Clin. Microbiol. Antimicrob. 15, 15. doi: 10.1186/s12941-016-0131-7

Hope, R., Warner, M., Mushtaq, S., Ward, M. E., Parsons, T., and Livermore, D. M. (2005). Effect of medium type, age and aeration on the MICs of tigecycline and classical tetracyclines. J. Antimicrob. Chemother. 56, 1042-1046. doi: $10.1093 /$ jac/dki386

Horiyama, T., Nikaido, E., Yamaguchi, A., and Nishino, K. (2011). Roles of Salmonella multidrug efflux pumps in tigecycline resistance. J. Antimicrob. Chemother. 66, 105-110. doi: 10.1093/jac/dkq421

Hornsey, M., Ellington, M. J., Doumith, M., Scott, G., Livermore, D. M., and Woodford, N. (2010). Emergence of AcrAB-mediated tigecycline resistance in 
a clinical isolate of Enterobacter cloacae during ciprofloxacin treatment. Int. J. Antimicrob. Agents 35, 478-481. doi: 10.1016/j.ijantimicag.2010.01.011

Hung, K. H., Wang, M. C., Huang, A. H., Yan, J. J., and Wu, J. J. (2012). Heteroresistance to cephalosporins and penicillins in Acinetobacter baumannii. J. Clin. Microbiol. 50, 721-726. doi: 10.1128/JCM.05085-11

Jenner, L., Starosta, A. L., Terry, D. S., Mikolajka, A., Filonava, L., Yusupov, M., et al. (2013). Structural basis for potent inhibitory activity of the antibiotic tigecycline during protein synthesis. Proc. Natl. Acad. Sci. U.S.A. 110, 3812-3816. doi: 10.1073/pnas.1216691110

Jiménez-Castellanos, J. C., Wan Ahmad Kamil, W. N., Cheung, C. H., Tobin, M. S., Brown, J., Isaac, S. G., et al. (2016). Comparative effects of overproducing the AraC-type transcriptional regulators MarA, SoxS, RarA and RamA on antimicrobial drug susceptibility in Klebsiella pneumoniae. J. Antimicrob. Chemother. 71, 1820-1825. doi: 10.1093/jac/dkw088

Keeney, D., Ruzin, A., and Bradford, P. A. (2007). RamA, a transcriptional regulator, and $\mathrm{Acr} A B$, an RND-type efflux pump, are associated with decreased susceptibility to tigecycline in Enterobacter cloacae. Microb. Drug Resist. 13, 1-6. doi: $10.1089 / \mathrm{mdr} .2006 .9990$

Keeney, D., Ruzin, A., McAleese, F., Murphy, E., and Bradford, P. A. (2008). MarA-mediated overexpression of the AcrAB efflux pump results in decreased susceptibility to tigecycline in Escherichia coli. J. Antimicrob. Chemother. 61, 46-53. doi: 10.1093/jac/dkm397

Kehrenberg, C., Cloeckaert, A., Klein, G., and Schwarz, S. (2009). Decreased fluoroquinolone susceptibility in mutants of Salmonella serovars other than Typhimurium: detection of novel mutations involved in modulated expression of ramA and soxS. J. Antimicrob. Chemother. 64, 1175-1180. doi: $10.1093 / \mathrm{jac} / \mathrm{dkp} 347$

Lee, H. Y., Chen, C. L., Wang, S. B., Su, L. H., Chen, S. H., Liu, S. Y., et al. (2011). Imipenem heteroresistance induced by imipenem in multidrugresistant Acinetobacter baumannii: mechanism and clinical implications. Int. J. Antimicrob. Agents 37, 302-308. doi: 10.1016/j.ijantimicag.2010.12.015

Li, C., Sutherland, C. A., Nightingale, C. H., and Nicolau, D. P. (2004). Quantitation of tigecycline, a novel glycylcycline [corrected] by liquid chromatography. J. Chromatogr. B Analyt. Technol. Biomed. Life Sci. 811, 225-229. doi: 10.1016/S1570-0232(04)00745-7

Li, L., Liao, X., Yang, Y., Sun, J., Li, L., Liu, B., et al. (2013). Spread of oqxAB in Salmonella enterica serotype Typhimurium predominantly by IncHI2 plasmids. J. Antimicrob. Chemother. 68, 2263-2268. doi: 10.1093/jac/dkt209

Liu, Y.-Y., Wang, Y., Walsh, T. R., Yi, L.-X., Zhang, R., Spencer, J., et al. (2016). Emergence of plasmid-mediated colistin resistance mechanism MCR-1 in animals and human beings in China: a microbiological and molecular biological study. Lancet Infect. Dis. 16, 161-168. doi: 10.1016/S1473-3099(15)00424-7

Moore, M. R., Perdreau-Remington, F., and Chambers, H. F. (2003). Vancomycin treatment failure associated with heterogeneous vancomycin-intermediate Staphylococcus aureus in a patient with endocarditis and in the rabbit model of endocarditis. Antimicrob. Agents Chemother. 47, 1262-1266. doi: 10.1128/AAC.47.4.1262-1266.2003

Morand, B., and Mühlemann, K. (2007). Heteroresistance to penicillin in Streptococcus pneumoniae. Proc. Natl. Acad. Sci. U.S.A. 104, 14098-14103. doi: 10.1073/pnas.0702377104

Nikaido, E., Yamaguchi, A., and Nishino, K. (2008). AcrAB multidrug efflux pump regulation in Salmonella enterica serovar Typhimurium by RamA in response to environmental signals. J. Biol. Chem. 283, 24245-24253. doi: $10.1074 /$ jbc.M804544200

Olliver, A., Vallé, M., Chaslus-Dancla, E., and Cloeckaert, A. (2004). Role of an acrR mutation in multidrug resistance of in vitro-selected fluoroquinolone-resistant mutants of Salmonella enterica serovar Typhimurium. FEMS Microbiol. Lett. 238, 267-272. doi: 10.1016/j.femsle.2004.07.046

Ong, C. T., Babalola, C. P., Nightingale, C. H., and Nicolau, D. P. (2005). Penetration, efflux and intracellular activity of tigecycline in human polymorphonuclear neutrophils (PMNs). J. Antimicrob. Chemother. 56, 498-501. doi: 10.1093/jac/dki260

Pérez, A., Poza, M., Aranda, J., Latasa, C., Medrano, F. J., Tomás, M., et al. (2012). Effect of transcriptional activators SoxS, RobA, and RamA on expression of multidrug efflux pump AcrAB-TolC in Enterobacter cloacae. Antimicrob. Agents Chemother. 56, 6256-6266. doi: 10.1128/AAC.01085-12

Pournaras, S., Kristo, I., Vrioni, G., Ikonomidis, A., Poulou, A., Petropoulou, D., et al. (2010). Characteristics of meropenem heteroresistance in Klebsiella pneumoniae carbapenemase (KPC)-producing clinical isolates of $\mathrm{K}$. pneumoniae. J. Clin. Microbiol. 48, 2601-2604. doi: 10.1128/JCM.02134-09

Ruzin, A., Keeney, D., and Bradford, P. A. (2007). AdeABC multidrug efflux pump is associated with decreased susceptibility to tigecycline in Acinetobacter calcoaceticus-Acinetobacter baumannii complex. J. Antimicrob. Chemother. 59, 1001-1004. doi: 10.1093/jac/dkm058

Sánchez-Romero, M. A., and Casadesús, J. (2014). Contribution of phenotypic heterogeneity to adaptive antibiotic resistance. Proc. Natl. Acad. Sci. U.S.A. 111, 355-360. doi: 10.1073/pnas.1316084111

Smith, S. I., Seriki, A., and Ajayi, A. (2016). Typhoidal and non-typhoidal Salmonella infections in Africa. Eur. J. Clin. Microbiol. Infect. Dis. 35, 1913-1922. doi: 10.1007/s10096-016-2760-3

Stein, G. E., and Babinchak, T. (2013). Tigecycline: an update. Diagn. Microbiol. Infect. Dis. 75, 331-336. doi: 10.1016/j.diagmicrobio.2012.12.004

Sun, Y., Dai, M., Hao, H., Wang, Y., Huang, L., Almofti, Y. A., et al. (2011). The role of RamA on the development of ciprofloxacin resistance in Salmonella enterica serovar Typhimurium. PLOS ONE 6:e23471. doi: 10.1371/journal.pone.00 23471

Taneja, N., and Kaur, H. (2016). Insights into Newer Antimicrobial Agents Against Gram-negative Bacteria. Microbiol. Insights 9, 9-19. doi: 10.4137/MBI. S29459

Tang, H. J., Chen, C. C., and Ko, W. C. (2016). Tigecycline therapy for bacteremia and aortitis caused by Salmonella enterica serotype Choleraesuis: a case report. J. Microbiol. Immunol. Infect. 49, 131-133. doi: 10.1016/j.jmii.2012.01.011

Veleba, M., De Majumdar, S., Hornsey, M., Woodford, N., and Schneiders, T. (2013). Genetic characterization of tigecycline resistance in clinical isolates of Enterobacter cloacae and Enterobacter aerogenes. J. Antimicrob. Chemother. 68, 1011-1018. doi: 10.1093/jac/dks530

Veleba, M., Higgins, P. G., Gonzalez, G., Seifert, H., and Schneiders, T. (2012). Characterization of RarA, a Novel AraC family multidrug resistance regulator in Klebsiella pneumoniae. Antimicrob. Agents Chemother. 56, 4450-4458. doi: 10.1128/AAC.00456-12

Wong, M. H., Chan, E. W., and Chen, S. (2015). Evolution and dissemination of OqxAB-like efflux pumps, an emerging quinolone resistance determinant among members of Enterobacteriaceae. Antimicrob. Agents Chemother. 59, 3290-3297. doi: 10.1128/AAC.00310-15

Yao, X., Doi, Y., Zeng, L., Lv, L., and Liu, J.-H. (2016). Carbapenem-resistant and colistin-resistant Escherichia coli co-producing NDM-9 and MCR-1. Lancet Infect. Dis. 16, 288-289. doi: 10.1016/S1473-3099(16)00057-8

Zheng, J., Cui, S., and Meng, J. (2009). Effect of transcriptional activators RamA and SoxS on expression of multidrug efflux pumps AcrAB and AcrEF in fluoroquinolone-resistant Salmonella Typhimurium. J. Antimicrob. Chemother. 63, 95-102. doi: 10.1093/jac/dkn448

Zheng, J., Tian, F., Cui, S., Song, J., Zhao, S., Brown, E. W., et al. (2011). Differential gene expression by RamA in ciprofloxacin-resistant Salmonella Typhimurium. PLoS ONE 6:e22161. doi: 10.1371/journal.pone.0022161

Zhong, X., Xu, H., Chen, D., Zhou, H., Hu, X., and Cheng, G. (2014). First emergence of $\mathrm{acr} A \mathrm{~B}$ and oqx $\mathrm{AB}$ mediated tigecycline resistance in clinical isolates of Klebsiella pneumoniae pre-dating the use of tigecycline in a Chinese hospital. PLoS ONE 9:e115185. doi: 10.1371/journal.pone.0115185

Conflict of Interest Statement: The authors declare that the research was conducted in the absence of any commercial or financial relationships that could be construed as a potential conflict of interest.

Copyright $\odot 2017$ Chen, Hu, Zhang, Liao, Liu and Sun. This is an open-access article distributed under the terms of the Creative Commons Attribution License (CC BY). The use, distribution or reproduction in other forums is permitted, provided the original author(s) or licensor are credited and that the original publication in this journal is cited, in accordance with accepted academic practice. No use, distribution or reproduction is permitted which does not comply with these terms. 